\title{
A longer 'tail' of repression
}

MicroRNAs (miRNAs) more strongly repress the translation of polyadenylated mRNAs than that of non-adenylated counterparts. A new study shows that this is because poly(A)-binding protein (PABP) promotes the association of miRNA-induced silencing complex (miRISC) with target mRNAs. miRNAs regulate gene expression by binding to partially complementary sites on their target mRNAs, which, in most cases, results in either a block in translation or mRNA destabilization and degradation. miRNAs can induce deadenylation of target mRNAs, and PABP is known to be required for this process. However, whether PABP has additional functions in miRNA-mediated repression other than promoting deadenylation was unclear.

Hentze and colleagues used a Drosophila melanogaster cell-free translation system to investigate the role of $\mathrm{PABP}$ and the poly(A) tail in miRNA-mediated repression. They first observed that mRNAs containing miRNA-binding sites in their 3 ' untranslated region
(3' UTR) were efficiently repressed when they had a poly(A) tail, whereas lack of a tail decreased miRNA-mediated repression. Furthermore, the authors observed greater repression of mRNAs with longer tails. Importantly, they found that mRNAs were repressed to the same extent in the absence of PABP regardless of whether they had a poly(A) tail. This suggests that PABP contributes to miRNA-mediated repression of polyadenylated mRNAs.

Next, by analysing the association of target mRNAs (those carrying miRNA-binding sites) with PABP by co-immunoprecipitation, the authors found that PABP is displaced from repressed $m$ RNAs early during miRNA-mediated translation repression. Thus, PABP at poly(A) tails might exert its function during the initial phases of silencing.

The authors went on to test whether $\mathrm{PABP}$ and poly(A) tails might influence the binding of miRISC, which is guided to its targets by
miRNAs and inhibits translation by binding to imperfectly matched sequences in the $3^{\prime}$ UTR of target mRNAs. Co-immunoprecipitation studies showed that the association of Argonaute 1 (AGO1), which is the miRNA-binding subunit of miRISC, with mRNAs was considerably lower in the absence of poly(A) tails. Furthermore, the AGO1-mRNA association was comparably low for mRNAs with or without a poly(A) tail when PABP was removed. Thus, PABP stimulates the binding of miRISC to polyadenylated mRNAs, and this was shown to occur very early in the establishment of repression. Interestingly, the recruitment of miRISC promoted the release of PABP, and this occurred prior to deadenylation.

This work reveals a new aspect of the interplay between PABP, poly(A) tails and miRNA-mediated silencing. The authors propose a model in which PABP and poly $(A)$ tails enhance the recruitment of miRISC to target mRNAs during early phases of silencing, which can then promote PABP displacement and deadenylation, thus leading to the establishment of effective silencing. Kim Baumann

ORIGINAL RESEARCH PAPER Moretti, F. et al. PABP and the poly(A) tail augment microRNA repression by facilitated miRISC binding. Nature Struct. Mol. Biol. 27 May 2012 (doi:10.1038/nsmb.2309) 